\title{
Kinase activity-tagged western blotting assay
}

\author{
Masumi Eto*,1, Shuichi Katsuki', Yoshinori Tanaka' \& Kosuke Takeya'
}

\begin{abstract}
Determining cellular activities of protein kinases is a fundamental step for characterizing pathophysiological cell signaling pathways. Here, we optimized a nonradioactive method that detects protein kinases in tissues or cells after separation by SDS-PAGE and transfer onto polyvinylidene fluoride membranes. The method, kinase activity-tagged western blotting (KAT-WB), consists of five steps: electrophoresis of cell extracts that contain protein kinases, electroblotting proteins onto polyvinylidene fluoride membrane, denaturation-renaturation, phosphorylation, with or without an added substrate protein and immunodetection using anti-phospho-specific antibodies. KAT-WB detected autophosphorylation of one Tyr-kinase and site-specific phosphorylation of added substrate by multiple kinases. KAT-WB assay enables us to interrogate multiple kinase signaling pathways without using radioactive ATP.
\end{abstract}

\section{METHOD SUMMARY}

Kinase activity-tagged western blotting is designed to separate and detect multiple kinases that phosphorylate themselves or a specific substrate. Kinases on the blot are subjected to denaturation with guanidine and then stepwise renaturation, prior to phosphorylation using nonradioactive ATP and detection with phosphospecific antibodies.

\section{KEYWORDS}

cell signaling $\cdot$ laboratory safety $\cdot$ nonradioactive assay $\cdot$ phosphorylation $\cdot$ posttranscriptional modification $\cdot$ protein kinases $\cdot$ western blotting

${ }^{1}$ Biochemistry Unit, Faculty of Veterinary Medicine, Okayama University of Science, Imabari, Ehime 794-8555, Japan; *Author for correspondence: m-eto@vet.ous.ac.jp

BioTechniques 68: 211-213 (April 2020) 10.2144/ btn-2019-0136

\section{GRAPHICALABSTRACT}

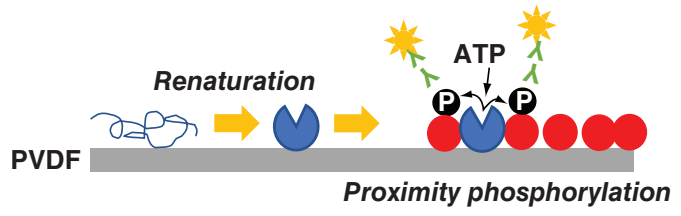

In KAT WB, denaturated kinases on PVDF membrane undergo renaturation and phosphorylate a specific substrate in the proximity to the kinase. Phosphorylation is detected using anti-phosphospecific antibodies.

Accumulating evidence suggests that disruptions in phosphorylation/dephosphorylation signaling are associated with pathological behaviors of cells. Phosphoproteome projects have identified nearly 300,000 phosphorylated Ser/Thr/Tyr residues in cellular proteins [1]. In parallel, the human genome project uncovered approximately 500 protein kinases. Yet, a limited number of protein kinases have been assigned to each phosphorylation site.

The in-gel phosphorylation assay has been a convenient method for detecting protein kinase activities in samples solubilized with SDS. This method was applied for identification of CaMKII, MYPT1-K (or ZIPK) and others [2-7]. In this assay protocol, protein kinases are loaded onto an SDS-polyacrylamide gel embedded with a substrate polypeptide, and then subjected to renaturation, phosphorylation using radioactive ATP, followed by autoradiography to detect phosphorylated substrate. In addition, autophosphorylation of kinases has been detected on nitrocellulose or polyvinylidene fluoride (PVDF) membrane using radioactive ATP [8]. The radioactive material may be a limitation for users, owing to safety regulations. In addition, autoradiograms of phosphorylated proteins lack any information of phosphorylation sites.

Here, we optimized a nonradioactive alternative assay to the traditional in-gel phosphorylation assay. Named kinase activity-tagged western blotting (KAT-WB), this successfully detects Tyr-kinases that autophosphorylate themselves as well as Ser/Thr-kinases that phosphorylate a substrate protein of interest at a specific site using phospho (P)-specific antibodies.

Figure 1 shows KAT-WB data for Tyr-kinase autophosphorylation assay. To make duplicate blots, two aliquots of an extract were loaded onto an SDS polyacrylamide gel, subjected to electrophoresis, and then proteins were transferred onto a sheet of PVDF membrane $(0.2 \mu \mathrm{m})$. The membrane was sequentially treated with buffered 2-propanol, 6.0 M guanidine hydrochloride $(\mathrm{Gu}-\mathrm{HCl}), 3.0 \mathrm{M}$ $\mathrm{Gu}-\mathrm{HCl}, 0.1 \mathrm{M} \mathrm{Gu}-\mathrm{HCl}$ and renaturation buffer (see Supplementary Protocol). After an overnight renaturation step at $4^{\circ} \mathrm{C}$, the membrane was cut lengthwise to generate two duplicate pieces (Figure 1). One piece of membrane was incubated for $60 \mathrm{~min}$ with $1 \mathrm{mM}$ MgATP. The other piece of membrane was incubated without MgATP, as a control. Both sheets were simultaneously subjected to immunodetection with anti-P-Tyr antibody (Clone PY20 at 1:5000 dilution). As shown in Figure 1, multiple P-Tyr bands were detected at 100, 70, 45 and $35 \mathrm{kDa}$ in the control membrane (top left). These P-Tyr proteins were presumably already phosphorylated prior to extraction. The incubation with MgATP after separation elevated Tyr phosphorylation of a protein at $45 \mathrm{kDa}$ in cardiac muscle lysates (Figure 1, top right, lane 2), indicating autophosphorylation of Tyr residues in this kinase on the membrane following renaturation. Figure 1 shows densitograms of lane 2 (-/+ MgATP). To verify equivalent loading of protein on the two blots, the filters were subjected to Coomassie staining (Figure 1, bottom). A technical note is that, in the course of developing the method, we found that the treat- 
1: Skeletal muscle

2: Cardiac muscle

3: Neuro2a

- ATP + ATP

$\begin{array}{lll:lll}1 & 2 & 3 & 1 & 2 & 3\end{array}$

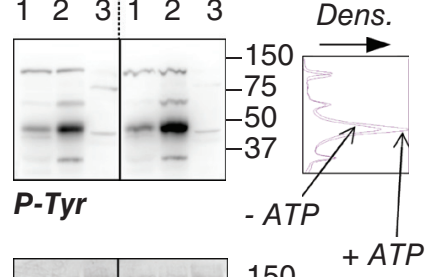

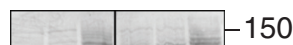

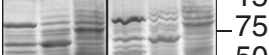

$-75$

$-37$

Coomassie

ments with buffered 2-propanol and 6M $\mathrm{Gu}-\mathrm{HCl}$ were both necessary for the detection of kinase activities. Therefore, KAT-WB detected a 45-kDa Tyr kinase.

Another version of KAT-WB uses sitespecific phosphorylation of a substrate protein absorbed on PVDF membrane in proximity to the kinase. We used phosphatase holoenzyme inhibitor-1 (PHI-1) as a protein of interest, whose phosphorylation at Thr57 converts this cellular protein into an inhibitor of type-1 Ser/Thr phosphatase [9]. Figure 2A shows KAT-WB data using as a positive control recombinant ZIPK, a Ser/ Thr kinase known to phosphorylate recombinant PHI-1 at Thr57 [10]. After the denaturation and the stepwise renaturation process, the membrane was soaked overnight at $4^{\circ} \mathrm{C}$ in the renaturation buffer supplemented with recombinant $\mathrm{PHI}-1$ wild type (WT) or an nonphosphorylatable version $(\mathrm{T} 57 \mathrm{H})$ at $0.2 \mathrm{mg} / \mathrm{ml}$, and then the phosphorylation reaction was run with MgATP. Phosphory-
Figure 1. Kinase activity-tagged-western blotting for autophosphorylation of Tyr-kinases. Total proteins of mouse skeletal muscle $(31 \mu \mathrm{g})$, cardiac muscle $(28 \mu \mathrm{g})$ and Neuro2a cells $(27 \mu \mathrm{g})$ were subjected to KAT-western blotting. After renaturation, the membrane was cut lengthwise into two, and each part was incubated for $60 \mathrm{~min}$ at $37^{\circ} \mathrm{C}$ in the absence (left) or presence (right) of $1 \mathrm{mM}$ ATP. Tyr-phosphorylation was detected by a conventional electrochemiluminescent method using anti-P-Tyr antibody (top). After the immunoblotting, total proteins on each blot were visualized by Coomassie staining (bottom). Numbers at right indicate molecular weights of marker proteins (in $\mathrm{kDa}$ ). A box to the right indicates densitograms of lane 2 in left and right panels. Levels of brightness and contrast of blots are identical.

lated $\mathrm{PHI}-1$ on the membrane was detected using anti-P-PHI-1 (Thr57) antibody [11], and this revealed a band of the recombinant ZIPK. However, only a faint signal was detected on the control membranes without PHI-1 or with the $\mathrm{T} 57 \mathrm{H}$ version of PHI-1. These data suggest that renaturated kinases on the membrane are able to phosphorylate substrates that are also absorbed onto the membrane. KAT-WB was applied to detect multiple PHI-1 kinases in tissues and cells (Figure 2B). Extracts from rat skeletal, cardiac muscles and Neuro2a cells were subjected to KAT-WB assay. Compared with no-ATP control (left, top), signals of P-PHI-1 appeared on the blot incubated with $1 \mathrm{mM}$ MgATP (right, top). Prominent bands of P-PHI-1 were detected in Neuro2a cell lysates at 100,60 and $49 \mathrm{kDa}$ (lane 3, open arrowhead). However, no bands for P-PHI-1 were evident in the lane with the extracts of skeletal and cardiac muscles (lanes 1 and 2). The P-PHI-1 signal was not enhanced by the phosphory- lation step without PHI-1 (Figure 2, bottom, blank: BLK). These results show that there are multiple PHI-1 kinases expressed in neuronal cells but not in skeletal or cardiac muscles. This provides us with a powerful tool for detecting multiple substrate-specific kinases in cells and tissues. A technical note is that the overnight renaturation with $\mathrm{PHI}-1$ was necessary for detection of the phosphorylation signals, and that a higher concentration of $\mathrm{PHI}-1$ did not increase the staining intensity (not shown).

Based on these data, we propose that KAT-WB is capable of detecting autophosphorylation of Tyr kinases and site-specific phosphorylation of selective substrate proteins of interest by multiple protein kinases present in tissues and cells. KAT-WB essentially shares advantages of the traditional in-gel phosphorylation assay, which detects kinase activities in a crude mixture, such as cell extracts, exposing their molecular sizes [7]. Another advantage of these two assays is that post-translational modifications (PTMs) of kinases in cells can be preserved at a desired time point under specific conditions, because the samples for these assays are quickly denatured with the buffer, including SDS, eliminating phosphorylation and dephosphorylation in the extracts [12]. Most protein kinases are regulated through PTM. KAT-WB is capable of monitoring fluctuations in kinase activities, even if regulatory PTMs have yet to be characterized. KAT-WB is unable to detect every kinase in cell extracts because only monomeric enzymes that renature effectively under the conditions employed show activity. However, modifications of KAT-WB should be able to detect kinases regulated
(A)

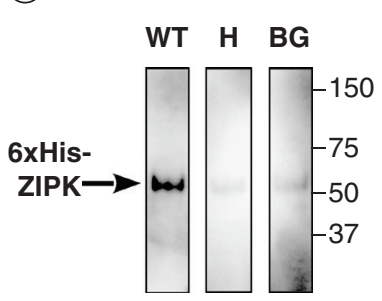

(B) 1: Skeletal muscle 2: Cardiac muscle 3: Neuro2a

- ATP + ATP

$\begin{array}{llllll}1 & 2 & 3 & 1 & 2 & 3\end{array}$
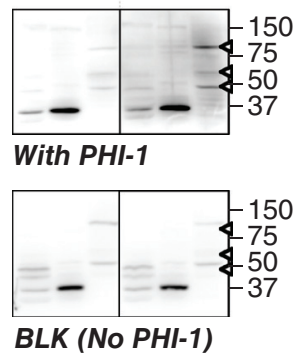

Figure 2. Kinase activity-tagged-western blotting for phosphorylation of a specific substrate protein. (A) Triplicate blots were prepared using recombinant ZIPK partially purified from lysates of cells expressing a $6 \times$ His-tagged version. After electroblotting and the denaturation-renaturation process, the membrane was incubated overnight with recombinant $\mathrm{PHI}-1$ proteins (WT: left; $\mathrm{T} 57 \mathrm{H}$ : center) at $0.2 \mathrm{mg} / \mathrm{ml}$ or without PHI-1 (BG). Each membrane was then incubated with $1 \mathrm{mM}$ ATP at $37^{\circ} \mathrm{C}$ and subjected to immunodetection using anti-P-PHI-1 (T57) antibody. (B) Two duplicate blots (top and bottom) were prepared using total proteins samples, as described in Figure 1. Recombinant PHI-1 $(0.2 \mathrm{mg} / \mathrm{ml})$ was added to the renaturation buffer for a blot (top). BLK indicates the blot without the recombinant PHI-1 treatment. After incubation in the absence (left) and presence (right) of $1 \mathrm{mM}$ ATP, the blots were subjected to immunodetection using anti-P-PHI-1 (Thr57). Arrowheads to the right indicate the locations of P-PHI-1 in top right panel. Levels of brightness and contrast of blots are identical. BG: Background; WT: Wild-type. 
by calmodulin and cyclins, as it has been done by in-gel phosphorylation assay $[4,5]$. Improvement of renaturation efficiency and a combination with immunoprecipitation/immunodepletion with antibodies for kinases may expand the utility of the approach.

It is noteworthy that, in addition to phosphorylation, the principle of KAT-WB protocol can be applied for detection of other PTM enzymes, such as acetylases, methylases and ubiquitin ligases, when PTM-specific antibodies are available. Along with the development of antibodies that recognize site-specific PTMs, research targets covered by activity-tagged western blotting will be expanded.

\section{AUTHOR CONTRIBUTIONS}

M Eto, S Katsuki, Y Tanaka and K Takeya conceived and designed the experiments. S Katsuki, Y Tanaka and K Takeya conducted the assays. M Eto, S Katsuki, Y Tanaka and $\mathrm{K}$ Takeya performed the data analysis. M Eto, S Katsuki, Y Tanaka and K Takeya wrote the paper.

\section{ACKNOWLEDGMENTS}

The authors would like to thank DL Brautigan for proofreading of the manuscript and for valuable suggestions.

\section{FINANCIAL \& COMPETING INTERESTS DISCLOSURE}

This work is supported by JSPS KAKENHI (JP19K06573 and JP19H03125) and Society for Research on Umami Taste, funds from Imabari city and Ehime prefecture, and a gift from $\mathrm{H}$ Awagi. The authors have no other relevant affiliations or financial involvement with any organization or entity with a financial interest in or financial conflict with the subject matter or materials discussed in the manuscript apart from those disclosed.

No writing assistance was utilized in the production of this manuscript.

\section{OPEN ACCESS}

This work is licensed under the AttributionNonCommercial-NoDerivatives 4.0 Unported License. To view a copy of this license, visit http://creativecommons.org/licenses/ by-nc-nd/4.0/

\section{REFERENCES}

1. Hornbeck PV, Zhang B, Murray B et al. PhosphoSitePlus, 2014: mutations, PTMs and recalibrations. Nucleic Acids Res. 43, D512-D520 (2015).

2. Celenza JL, Carlson M. Renaturation of protein kinase activity on protein blots. Methods Enzymol. Academic Press, Cambridge Massachusetts 34, 423-430 (1991)

3. Hutchcroft JE, Anostario M, Harrison ML et al. Renaturation and assay of protein kinases after electro- phoresis in sodium dodecyl sulfate-polyacrylamide gels. Methods Enzymol. Academic Press, Cambridge Massachusetts 33, 417-423 (1991).

4. Kameshita I, Fujisawa H. A sensitive method for detec tion of calmodulin-dependent protein kinase II activity in sodium dodecyl sulfate-polyacrylamide gel. Anal. Biochem. 183(1), 139-143 (1989).

5. Shackelford DA, Zivin JA. Renaturation of calcium/ calmodulin-dependent protein kinase activity after electrophoretic transfer from sodium dodecyl sulfate-polyacrylamide gels to membranes. Anal. Biochem. 211(1) 131-138 (1993).

6. Macdonald JA, Borman MA, Muranyi A et al. Identification of the endogenous smooth muscle myosin phosphatase-associated kinase. Proc. Natl Acad. Sci. USA 98(5), 2419-2424 (2001).

7. Wooten MW. In-gel kinase assay as a method to identify kinase substrates. Sci. STKE 2002(153), pl15 (2002).

8. Celenza J, Carlson M. A yeast gene that is essential for release from glucose repression encodes a protein kinase. Science 233(4769), 1175-1180 (1986).

9. Eto M, Karginov A, Brautigan DL. A novel phosphoprotein inhibitor of protein type-1 phosphatase holoenzymes. Biochemistry 38(51), 16952-16957 (1999).

10. Macdonald JA, Eto M, Borman MA et al. Dual Ser and Thr phosphorylation of CPI-17, an inhibitor of myosin phosphatase, by MYPT-associated kinase. FEBS Lett. 493(2-3), 91-94 (2001).

11. Eto $M$, Leach $C$, Tountas NA et al. Phosphoprotein inhibitors of protein phosphatase-1. Methods Enzymol. 366, 243-260 (2003).

12. Rousell J, Haddad El B, Lindsay MA et al. Regulation of $\mathrm{m} 2$ muscarinic receptor gene expression by platelet-derived growth factor: involvement of extracellular signal-regulated protein kinases in the down-regulation process. Mol. Pharmacol. 52(6), 966-973 (1997). 